\title{
Fault Detection and Diagnosis in Induction Machines: A Case Study
}

\author{
Miguel Marques ${ }^{1}$, João Martins ${ }^{2}$, V. Fernão Pires ${ }^{3,4}$, \\ Rui Dias Jorge ${ }^{5}$, and Luís Filipe Mendes ${ }^{5}$ \\ ${ }^{1}$ Department of Electrical and Computer Engineering, Faculty of Sciences and Technology - \\ University New of Lisbon, Quinta da Torre, 2829-516 Caparica, Portugal \\ ${ }^{2}$ CTS/UNINOVA, Faculty of Sciences and Technology - University New of Lisbon, Quinta da \\ Torre, 2829-516 Caparica, Portugal \\ ${ }^{3}$ Instituto Politécnico de Setúbal, Campus do IPS, Estefanilha, 2914-508 Setúbal, Portugal \\ ${ }^{4}$ Center for Innovation in Electrical and Energy Engineering (CIEEE), Lisbon, Portugal \\ ${ }^{5}$ EFACEC, Rua da Garagem, ${ }^{\circ}{ }^{\circ}$, Carnaxide, 2790-078, Oeiras, Portugal
}

\begin{abstract}
Nowadays in industry there many processes where human intervention is replaced by electrical machines, especially induction machines due to his robustness and performance. Although, induction machines are a high reliable device, they are also susceptible to faults. Therefore, the study of induction machine state is essential to reduce human and financial costs. It is presented in this paper an on-line system for detection and diagnosis of electrical faults in induction machines based on computer-aided monitoring of the supply currents. The main objective is to detect and identify the presence of broken rotor bars and stator short-circuits in the induction motor. The presence of faults in the machine causes different disturbances in the supply currents. Through a stationary reference frame, such as $\alpha \beta$-vector transform it is possible to extract and manipulate the results obtained from the supply currents using Principal Component Analysis (PCA).
\end{abstract}

Keywords: induction motor, diagnosis, fault detection, principal component analysis, PCA, eigenvalue, eigenvector.

\section{$1 \quad$ Introduction}

Three-phase induction machines perform critical functions as part of industrial processes. It is estimated that induction motors typically consume $40 \%$ to $50 \%$ of all electrical energy produced in a country [1]. Therefore, induction motors have a special role in the economy of industrialized countries.

Due to its importance, is urgent to develop intelligent systems that detect the presence of faults in the machines in order to reduce maintenance costs. These systems will allow the possibility of scheduled maintenance and predict the need for maintenance before serious deterioration or fault occurs, making it possible to increase the reliability of equipment, the improvement of his behavior and performance [2]. 
Concerning to maintenance of induction machines, preventive maintenance is widely used currently. It consists in periodic inspections with the objective of replacing parts that are supposed to break after a certain number of hours. However, even with periodic inspections this type of maintenance presents disadvantages such as, unneeded maintenance costs and in some situations catastrophic failures still likely to occur. Thus, the concept of condition-based maintenance (CBM) has emerged as an alternative to preventive maintenance. This type of maintenance is defined as the process of monitoring characteristics or parameters of a machine, in order to verify early changes and trends that can be used to indicate a fault situation or the need for maintenance.

In the last 20 years, FDD in induction machines is a research area that had a great evolution, as seen by the number of proposed methodologies, such as neural networks [3], current space patterns [4], fuzzy logic [5], spectral analysis [6] and vibration monitoring [7].

\section{Contribution to Internet of Things}

The paper presents the development of a commercial application for fault detection and diagnosis of electrical faults in induction machines. This analysis, based on PCA methodology [8,9], allows to conclude the practical feasibility of on-line monitoring through current space pattern analysis using an industrial product, such as the Terminal Protection Unit (TPU) developed by EFACEC. In the future the use of condition-based systems, based on non-invasive measurements, will contribute to connect the induction machines with cyberspace making it possible to know the state of the machine remotely. Thus, since TPU allow the use of multiple communication standards it will be possible to make a remote monitoring and diagnosis of the devices connected to TPU.

\section{Principal Components Analysis (PCA)}

Principal Component Analysis is a non-parametric statistical method used to reduce the number of original variables, which are correlated, in a set of new uncorrelated variables referred as Principal Components (PC). The first public descriptions of this method were given in 1901 by Pearson [10] and latter developed in 1933 by Hotelling [11].

PCA can be obtained through several ways, such as eigenvalue decomposition of a matrix or single value decomposition (SVD) of a matrix [12]. In the case of eigenvalue decomposition it consists in the representation of matrix in terms of its eigenvalues and eigenvectors. Through the definition of eigenvectors, this technique is able to obtain the main directions of the data sample on a space-vector. It also possible to measure the weight of the sampled data spread through the main directions defined by the eigenvectors. These metric values are defined as eigenvalues [12].

Let $\mathrm{X} \in \mathfrak{R}^{n \times m}$ represents a data matrix, where $\mathrm{n}$ denotes the number of measurements and $\mathrm{m}$ denotes the number of physical variables. The $\mathrm{X}^{\mathrm{T}} \in \mathfrak{R}^{m \times n}$ 
represents the transposed matrix of $X$, where $m$ and $n$ have the same meaning as in the $X$ matrix. From the product of the two matrices $\mathrm{X}$ and $\mathrm{X}^{\mathrm{T}}$ is obtained a square matrix $\mathrm{E} \in \mathfrak{R}^{n \times n}$ called correlation matrix.

$$
\mathrm{E}=\mathrm{X}^{\mathrm{T}} \mathrm{X}
$$

After establishing the correlation matrix the eigenvectors and the respective eigenvalues, of $\mathrm{E}$ are calculated. There are several ways to define eigenvectors and eigenvalues, the most common approach defines an eigenvector of the matrix $\mathrm{E}$ as a vector that satisfies the following equation:

$$
(\mathrm{E}-\lambda \mathrm{I}) \mathrm{u}=0
$$

In fact, this concept of reducing the number of variables through PCA is useful in energy systems, particularly three-phase systems, such as three-phase induction machines. In three-phase energy systems without neutral connection it is usual to use the $\alpha \beta$-vector transformation to reduce the number of original variables. This transformation converts the three-phase currents or voltages into an equivalent twophase system. So in ideal conditions, the three-phase currents lead to $\alpha \beta$-vector with the following components:

$$
\left\{\begin{array} { l } 
{ \mathrm { i } _ { \alpha } = \sqrt { \frac { 2 } { 3 } } \mathrm { i } _ { \mathrm { a } } - \frac { 1 } { \sqrt { 6 } } \mathrm { i } _ { \mathrm { b } } - \frac { 1 } { \sqrt { 6 } } \mathrm { i } _ { \mathrm { c } } } \\
{ \mathrm { i } _ { \beta } = \frac { 1 } { \sqrt { 2 } } \mathrm { i } _ { \mathrm { b } } - \frac { 1 } { \sqrt { 2 } } \mathrm { i } _ { \mathrm { c } } }
\end{array} \Leftrightarrow \left\{\begin{array}{l}
\mathrm{i}_{\alpha}=\frac{\sqrt{6}}{2} \mathrm{I} \sin \omega \mathrm{t} \\
\mathrm{i}_{\beta}=\frac{\sqrt{6}}{2} \mathrm{I} \sin \left(\omega \mathrm{t}-\frac{\pi}{2}\right)
\end{array}\right.\right.
$$

Under normal conditions and with a balanced and constant frequency power supply, a pure sinusoidal signal makes a circular pattern centered at the origin of the $\alpha \beta$ coordinates. In Fig. 1 there is the representation of a healthy motor input current in the $\alpha \beta$-vector pattern.

However under abnormal conditions and considering a constant frequency power supply the previous conditions are no longer valid and the $\alpha \beta$ pattern loses its circular

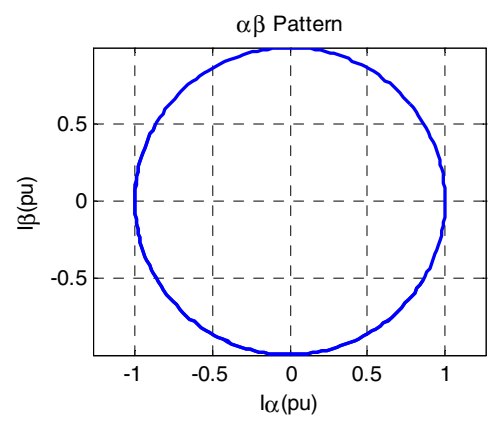

Fig. 1. Healthy motor input current $\alpha \beta$-vector pattern 
shape. For a situation where occurs a stator winding fault the input current $\alpha \beta$ pattern becomes an ellipse because there is an amplitude variation in the current of the winding that is in a fault situation. The patterns related to a stator winding fault are presented in the Fig. 2.
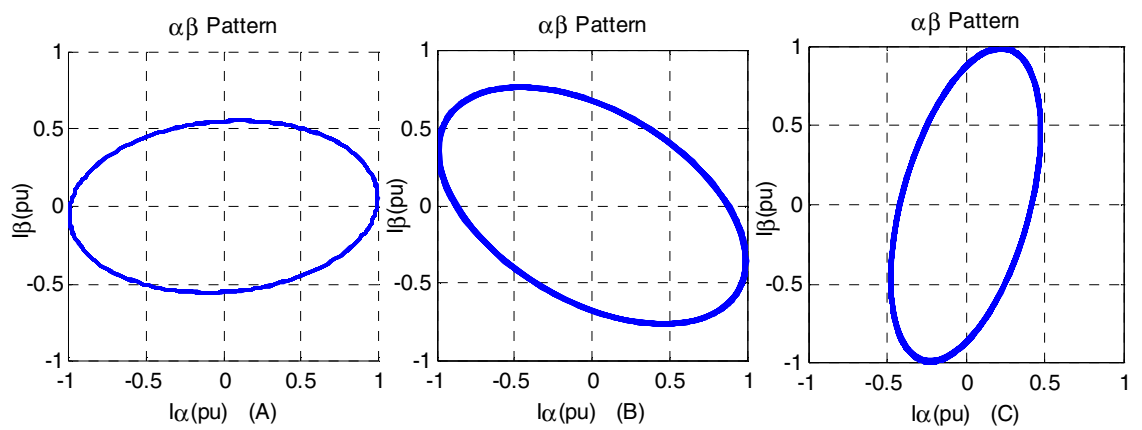

Fig. 2. Stator fault input current $\alpha \beta$-vector patterns. (A) stator fault in phase A (B) stator fault in phase $\mathrm{B}(\mathrm{C})$ stator fault in phase $\mathrm{C}$

When the motor presents a rotor fault situation the $\alpha \beta$-vector pattern presents a circular shape but the eigenvalues are not constant. It is possible to observe (Fig. 3) the appearance of a thick ring and the thickness of the ring increases with the severity of the fault. Cardoso et al. [13] concluded that the severity of the fault is proportional to the number of the rotor bars, but there is a moment where severity of the factor decreases as the number of broken bars increases.

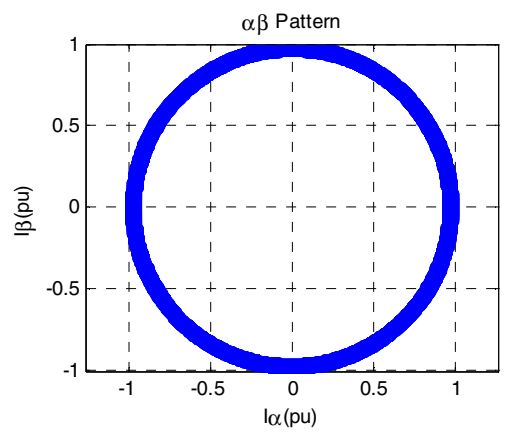

Fig. 3. Rotor fault input current $\alpha \beta$-vector pattern

\section{Experimental Results}

\subsection{Experimental Set Up}

The experimental set up used is depicted in Fig. 4. A series of tests were conducted on three squirrel-cage induction motors with a mechanical power $\left(\mathrm{P}_{\text {mec }}\right)$ of $2 \mathrm{Hp}, 230 / 400$ 
$\mathrm{V}$ nominal voltage $\left(\mathrm{V}_{\text {nom }}\right)$, a rated speed $(\mathrm{N})$ of $3000 \mathrm{rpm}$, all with same parameters. One motor was considered a healthy motor and tested. The other two motors were tested with stator short-circuits and broken rotor bars faults.

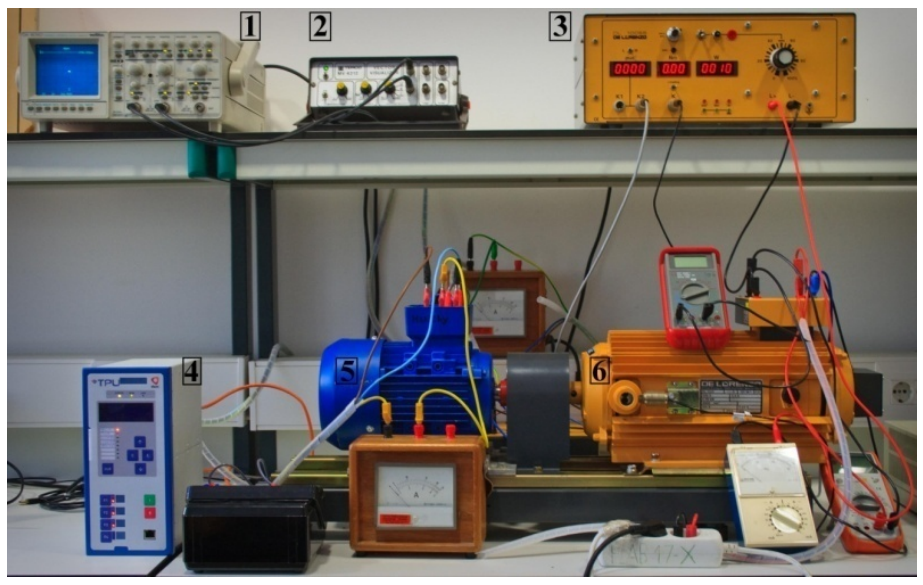

Fig. 4. Experimental apparatus used in this work

In the performed tests the motors $\{5\}$ were fed with a three-phase auto-transformer with an apparent power (S) of $4 \mathrm{kVA}$ and 0-400 $\mathrm{V}_{\mathrm{lnl}}$ (line-to-line). The mechanical load was applied to the induction motor by connecting the shaft to a DC generator $\{6\}$ (controlled by a dc current source $\{3\}$ ) of $0.75 \mathrm{~kW}$ rated power $\left(\mathrm{P}_{\mathrm{el}}\right)$. The output of the DC generator was connected to a variable resistive load. In order to allow tests to be performed at different load levels, the DC excitation current and the load resistor were both adjustable.

The data acquisition, signal conditioning and data processing are performed by the TPU S220 \{4\} developed by EFACEC. For the laboratory tests, a broken rotor bar fault was introduced by drilling a hole into a bar, the hole diameter is slightly larger than the bar width. In the case of short-circuits in the stator windings they were applied by introducing an external variable resistor in series with the windings of each phase.

\subsection{Experimental Results}

The motor was initially tested in a healthy situation, with the stator windings and its cage intact, in order to verify the current $\alpha \beta$-vector reference pattern. In the conducted tests were applied various levels torque, in order to verify the robustness of the algorithm at different load levels. The start-up condition was discarded since it is not considered by the algorithm.

The temporal evolution of the motor stator currents is represented in the Fig. 5 (A). The Fig. 5 (B) does not present the $\alpha \beta$-vector with a circular shape due to experimental limitation, such as harmonic distortion in the supply voltage and the magnetic field distribution in the machine is not perfectly sinusoidal. However the machine was considered in a healthy condition. For stator faults were used three 
variable resistors with $11.2 \Omega / 5 \mathrm{~A}$ in series with the impedance of each phase of the machine $(Z=4.8 \Omega)$. In the case of a fault severity factor of $60 \%$ the resistance value is $1.2 \Omega$. The $\alpha \beta$-vector (Fig. 6) no longer presents a "circular" shape and exhibit an elliptical shape.
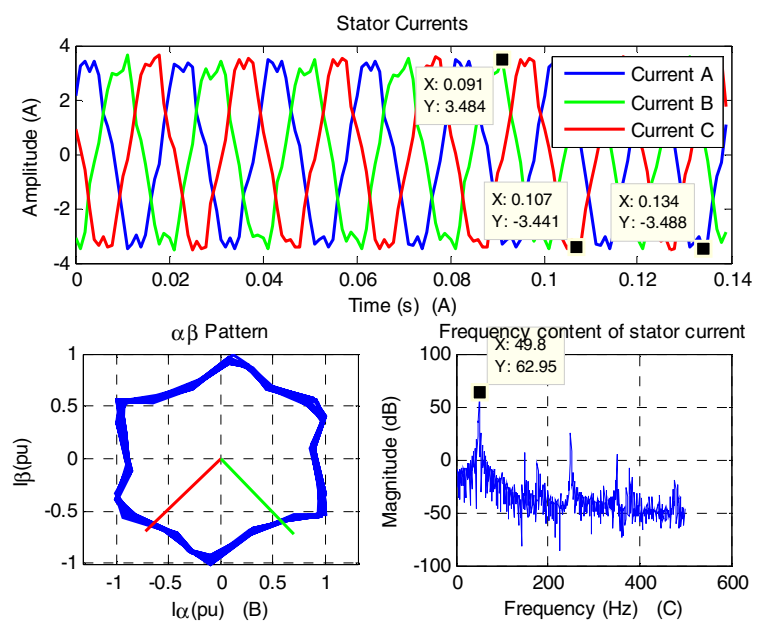

Fig. 5. (A) Stator currents of the machine in nominal operation (B) Experimental $\alpha \beta$-vector (C) Current A spectrum
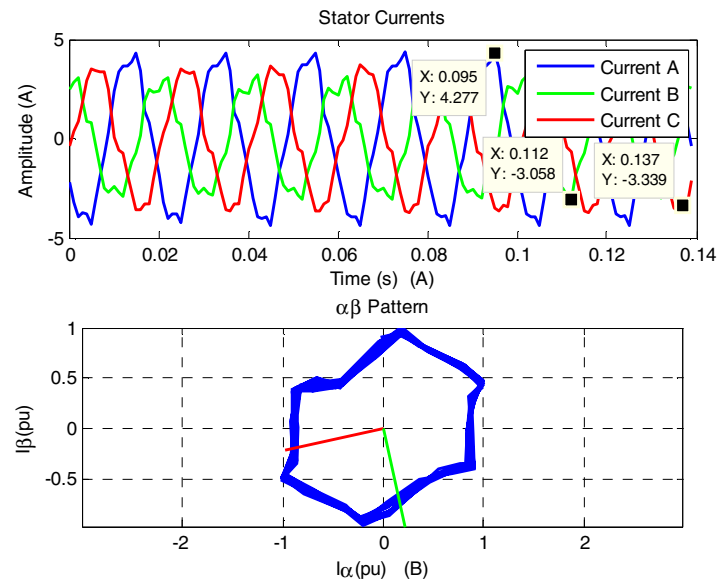

Fig. 6. Experimental results obtained for a stator fault situation in nominal operation with a SF $=60 \%$ in the phase A (A) Stator currents of the machine (B) Experimental $\alpha \beta$-vector

The results obtained for 6 broken rotor bars (major fault) are shown in the Fig. 8. The appearance of a thick ring (Fig. 8 (A)) in a rotor fault situation can be detected through the variation of eigenvalues (Fig. 8 (B)). In the experimental results the eigenvalues does not present a sinusoidal behavior, but show a periodic variation over time. 
The results obtained for 6 broken rotor bars (major fault) are shown in the Fig. 8. The appearance of a thick ring (Fig. 8 (A)) in a rotor fault situation can be detected through the variation of eigenvalues (Fig. 8 (B)). In the experimental results the eigenvalues does not present a sinusoidal behavior, but show a periodic variation over time.
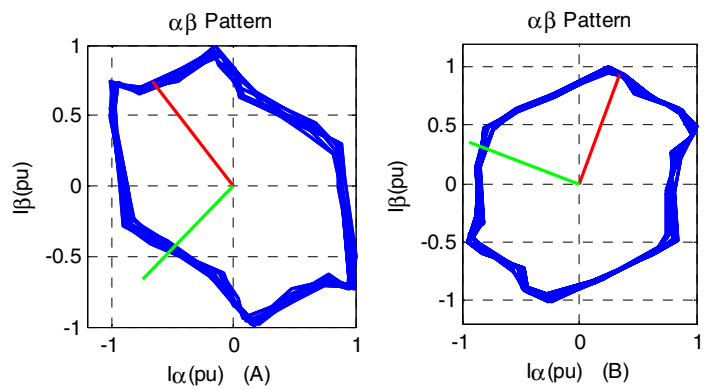

Fig. 7. Experimental $\alpha \beta$-vector pattern for a $\mathrm{SF}=60 \%$ (A) Phase $\mathrm{B}$ (B) Phase $\mathrm{C}$
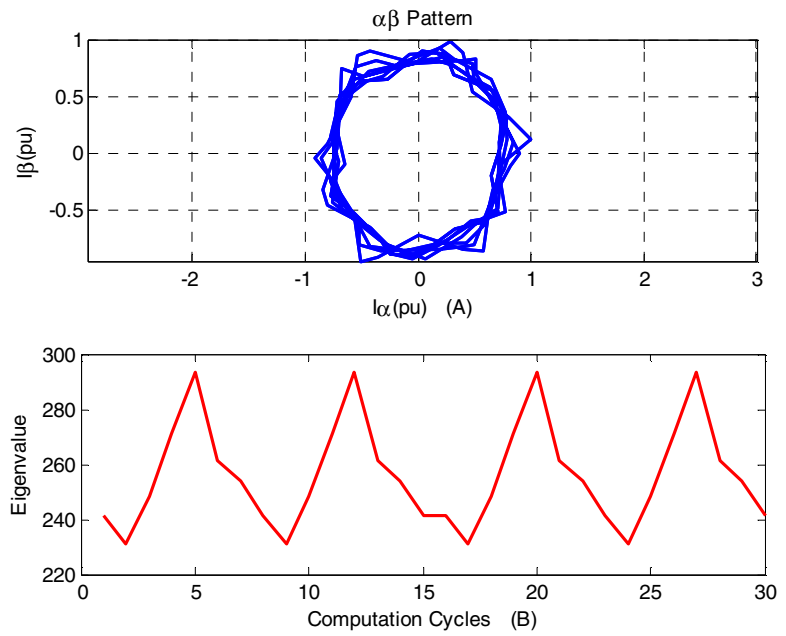

Fig. 8. Experimental results obtained for the machine with 6 broken rotor bars (A) Experimental $\alpha \beta$-vector pattern (B) Variation of the eigenvalues over the computation cycles

\section{$5 \quad$ Conclusions and Future Work}

The research in the field of fault detection and diagnosis could provide a reduction in operational costs and the increase of the machine's reliability that associated with remote systems will enable the interconnection of the induction machines with the cyberspace.

In this paper is presented an on-line fault detection and diagnosis system for threephase induction motors based on PCA. The proposed system uses the eigenvalue decomposition to detect changes in the $\alpha \beta$-vector pattern. 
The experimental results indicate that it is possible to detect the presence of shortcircuits in the stator windings and broken rotor bars in induction machines with this FDD method. Through the analysis of the eigenvalues from the $\alpha \beta$-vector components it is possible to infer if the machine is in a fault situation or not. For stator fault situations using the eigenvectors, it is also possible to identify in which phase the fault occurred.

Concerning to future works the stator faults were applied through the addition of variable resistors in series with the stator windings. This set up can be considered as an approximation to the fault situation. In the future should be short-circuited some stator windings and test the algorithm to observe his behavior.

Acknowledgements. Authors would like to thank to CTS of UNINOVA for the financial support and to EFACEC for providing its facilities and equipment for this work.

\section{References}

1. Ahmed, I., Supangat, R., Grieger, J., Ertugrul, N., Soong, W.L.: A Baseline Study for OnLine Condition Monitoring of Induction Machines. In: Australasian Universities Power Engineering Conference, pp. 26-29 (2004)

2. Siyambalapitiya, D., Mclaren, P.: Reliability Improvement and Economic Benefits of OnLine Monitoring Systems for Large Induction Machines. IEEE Transactions on Industry Applications 26(6), 1018-1025 (1990)

3. Chow, M., Mangum, P.: Neural Network Approach to Real-Time Condition Monitoring of Induction Motors. IEEE Transactions on Industry Electronics 38(6), 448-453 (1991)

4. Cardoso, A., Saraiva, E.: Computer Aided Detection of Airgap Eccentricity in Operating Three-phase Induction Motors, by Park's Vector Approach. IEEE Transactions on Industry Applications, 897-901 (1993)

5. Nejjari, H., Benbouzid, M.: Application of Fuzzy Logic to Induction Motors Condition Monitoring. IEEE Power Engineering Review, 52-54 (1999)

6. Thomson, W.: On-line MCSA to Diagnose Shorted Turns in Low Voltage Stator Windings of 3-Phase Induction Motors prior to Failure. In: IEEE International Electric Machines and Drives Conference, pp. 891-898 (2001)

7. Tsypkin, M.: Induction Motor Condition Monitoring: Vibration Analysis Technique - a Practical Implementation. In: IEEE International Electric Machines \& Drives Conference, pp. 406-411 (2011)

8. Martins, J.F., Pires, V.F., Amaral, T.: Induction motor fault detection and diagnosis using a current state space pattern recognition. Pattern Recognition Letters 32(2), 321-328 (2011)

9. Pires, V., Martins, J., Pires, A.: On-Line Diagnosis of Three-Phase Induction Motor Using an Eigenvalue $\alpha \beta$-vector approach. In: IEEE Conference on Power Electronics and Drive Systems, pp. 689-693 (2007)

10. Pearson, K.: On Lines and Planes of Closest Fit to Systems of Points in Space. Philosophical Maganize, 559-572 (1901)

11. Hotelling, H.: Analysis of a complex of statistical variables into principal components. Journal of Educational Psychology 24, 417-441 (1933)

12. Jolliffe, I.: Principal Component Analysis. Springer, New York (2002)

13. Cardoso, A., Cruz, S., Carvalho, J., Saraiva, E.: Rotor Cage Fault Diagnosis in ThreePhase Induction Motors, by Park's Vector Approach. In: IEEE Industry Applications Conference, vol. 1, pp. 642-646 (1995) 